\title{
Risk factors for viral hepatitis $C$ infection in Rwanda: results from a nationwide screening program
}

Jean Damascene Makuza ${ }^{1 *}$ (D, Carol Y. Liu², Corneille Killy Ntihabose ${ }^{1}$, Donatha Dushimiyimana ${ }^{1}$, Sabine Umuraza ${ }^{2}$, Marie Paul Nisingizwe ${ }^{3}$, Justine Umutesi ${ }^{1}$, Janvier Serumondo ${ }^{1}$, Soline Dusabeyesu Mugeni ${ }^{2}$, Muhamed Semakula ${ }^{1}$, Neil Gupta ${ }^{4}$, Margaret Hellard ${ }^{5}$ and Sabin Nsanzimana ${ }^{1}$

\begin{abstract}
Background: The epidemiology and risk factors for hepatitis C virus (HCV) infection in Rwanda are not well known; however, this information is crucial to shaping the country's public health approach to hepatitis $C$ control.

Methods: A HCV screening campaign was conducted in the general population in 24 districts previously identified to have a high HCV disease burden. At the time of sample collection, sociodemographic information and selfreported risk factors were collected. Bivariate and multivariate logistic regressions were conducted to assess risk factors independently associated with hepatitis $\mathrm{C}$ antibodies (HCVAb) seroprevalence.

Results: Out of a total of 326,263 individuals screened for HCVAb, 22,183 (6.8\%) were positive. In multivariate analysis, risk factors identified as statistically associated with HCVAb Seroprevalence include history of traditional operation or scarification $(\mathrm{OR}=1.09,95 \% \mathrm{Cl}: 1.05-1.14)$, presence of viral hepatitis in the family $(\mathrm{OR}=1.27,95 \% \mathrm{Cl}: 1.15-1.40)$, widowed or separated/ divorced ( $\mathrm{OR}=1.36,95 \% \mathrm{Cl}: 1.26-1.47)$, Southern province $(\mathrm{OR}=1.98,95 \% \mathrm{Cl}: 1.88-2.08)$ and aged 65 years and older $(\mathrm{OR}=4.86,95 \% \mathrm{Cl}: 4.62-5.11)$. Ubudehe category $3(\mathrm{OR}=0.97,95 \% \mathrm{Cl}: 0.93-1.01)$ and participants using RAMA (Health insurances for employees of public and private sectors) insurance $(\mathrm{OR}=0.76,95 \% \mathrm{Cl}: 0.70-0.85)$ had lower odds of HCV seroprevalence.
\end{abstract}

Conclusions: Our findings provide important information for Rwanda's strategy on prevention and case-finding. Future prevention interventions should aim to reduce transmission through targeted messaging around traditional healing practices and case-finding targeting individuals with a history of exposure or advanced age.

Keywords: Viral hepatitis C, Risk factors, Rwanda

\section{Background}

Globally, an estimated 71 million people are infected with chronic hepatitis $\mathrm{C}$ virus (HCV) infection [1]. Viral hepatitis contributed to 1.34 million deaths in 2015, a number comparable to annual deaths caused by tuberculosis and exceeding annual deaths caused by HIV. HCV accounts for around 400,000 deaths per year [2] and HCV-associated deaths in 2015 were mainly caused by chronic liver disease such as decompensated cirrhosis and liver cancer. The overall global HCV prevalence is estimated to be $2.5 \%$ and around $2.9 \%$ in Africa [3].

\footnotetext{
* Correspondence: makorofr@gmail.com

${ }^{1}$ IHDPC Department, Rwanda Biomedical Center, Po Box 7162, Kigali, Rwanda Full list of author information is available at the end of the article
}

While $\mathrm{HCV}$ is increasingly highlighted as an important contributor to disease burden in high-income countries such as Europe, Canada and the United States [4], the burden in the African region is less known and thought to be highly variable across geographic area [5]. The prevalence of $\mathrm{HCV}$ among the general population in Sub-Saharan African (SSA) ranges from 0.1 to $17.5 \%$, with countries such as Burundi (11.3\%) and Cameroon (13.8\%) among some of the countries with the highest prevalence in the world [6]. While increasing resources have been dedicated to address the burden of HCV in some high-income countries, to date, there remains a lack of strategic planning for prevention and management of $\mathrm{HCV}$ in SSA despite accumulating evidence of a

(c) The Author(s). 2019 Open Access This article is distributed under the terms of the Creative Commons Attribution 4.0 International License (http://creativecommons.org/licenses/by/4.0/), which permits unrestricted use, distribution, and 
significant disease burden [5]. The lack of a coordinated response among countries in SSA has further led to uncertainties on $\mathrm{HCV}$ prevalence and its variations across sociodemographic and geographic factors. Moreover, few studies in SSA have quantified the prevalence of past-exposures to known risk factors. The association between such risk factors and $\mathrm{HCV}$ infection and those studies were conducted only on specific groups, such as people living with HIV and MSM [7, 8] rather than the general population.

In Rwanda, the prevalence of HCV is not well known among the general population. Recent studies conducted in specific population groups have found the prevalence of anti-HCV (HCVAb), a marker for exposure to HCV, to be between $4.3-4.7 \%$ among people living with HIV (PLHIV) and 2.6\% among pregnant women $[9,10]$. Among these studies none have assessed risk factors for $\mathrm{HCV}$ in Rwanda.

In addition to uncertainties around HCVAb prevalence, risk factors for HCV infection in Rwanda have not been quantified on a national scale. Globally, older age, occupational risk of and being exposed to blood productsor individuals exposed to body piercings were shown to be risk factors for $\mathrm{HCV}$ [10-13]. In Africa, a systematic review yielded a wide range of high risk populations including, individuals infected with HIV, patients on hemodialysis, patients with history of blood transfusions, health care workers after needle stick injuries and sexually active adults with multiple partners [6].

Rwanda recently announced an ambitious campaign to eliminate $\mathrm{HCV}$. Understanding the $\mathrm{HCV}$ prevalence and current drivers of transmission will be crucial to guiding more efficient screening campaigns and implementing preventative activities to reduce population exposure to major routes of transmission. This study reports on the results from an $\mathrm{HCV}$ screening campaign conducted by the Government of Rwanda including the risk factors most likely contributing to the HCV Ab Seroprevalence in Rwanda.

\section{Methods}

\section{Study design}

The study consists of a secondary analysis of cross-sectional data collected during the 2018 screening campaign for HCV. All data used for analysis were collected at the time of viral hepatitis screening through use of a standardized laboratory request form which contained sociodemographic, comorbidities and viral hepatitis risk factors characteristics.

\section{Study population and recruitment of participants}

In response to the global and regional urgency to address $\mathrm{HCV}$ and improve understanding around $\mathrm{HCV}$ epidemiology, the Rwanda Biomedical Center (RBC) and partners prepared screening campaigns to identify infected patients to be linked to a free of charge treatment. In 2018, campaigns were specifically conducted in districts previously found to have higher prevalence of $\mathrm{HCV}$ among the general population and targeted individuals aged 25 years old and above from districts in Southern, Eastern and Northern Provinces previously found to have higher prevalence. The study population consists of voluntary participants of the 2018 campaigns. People who were not Rwandan by nationality or under 25 years old were excluded. Screening campaigns were conducted between March to October 2018 for 2 weeks in each district. Community awareness for viral hepatitis screening was done by radio advertisements and with the help of community health workers before and during the screening period. Individuals who belonged to targeted demographic groups and attended screening sites (health centers) were screened for HCV and included in the study population of this analysis.

\section{Data collection procedures}

Trained nurses and laboratory technicians, using a laboratory request form to record demographic, clinical, and behavior characteristics, collected samples after verbal consent of participants. Unlike previous campaigns, the 2018 campaign asked individuals about their exposure to a list of known risk factors for HCV including history of undergoing health treatments such as blood transfusions, surgical interventions and traditional operations or scarifications and comorbidities such as TB, diabetes and cancer. Testing was performed at 13 sites across Rwanda using Murex enzyme-linked immunosorbent assays (ELISA) for HCVAb (version 4.0; DiaSorin S.p.A, Italy). A team of laboratory technicians from the National Reference Laboratory supervised all testing activities. When laboratory results were available, the results and the contents of the lab request form were entered into an encrypted database (Microsoft Excel). The database was de-identified for the present study and no persons involved in the analysis of data were able to access the linked database.

\section{Variables}

The primary outcome of interest was HCVAb seroprevalence; it was a binary variable of either positive or negative as determined by the ELISA test. Independent variables included age. Age was considered as continuous variable during analysis then categorized into five groups: less than 35 years old, 35-44 years old, 45-54 years old, 55-64 years old and over 65 years old. Then sex, screening district, marital status and socioeconomic status (Ubudehe category). Ubudehe is a development programme whereby citizens are placed into different socioeconomic categories. The lowest socioeconomic 
category is 1 and the highest is 4 . Socioeconomic status was defined in accordance with the updated Ubudehe category by the Ministry of Local Government of Rwanda (MINALOC) [14]. The households that consume less than Rwandan Francs (RWF) 105,064 [equivalent to USD 120] per year are classified in Ubudehe Category 1 (poorest category), which includes people without houses and those affected by food insecurity. Households that consume between RWF 105,064 and RWF 159,375 (exclusively) [equivalent to USD 180] per year are in Ubudehe Category 2, which includes households subsisting on manual labor or temporary employment and those capable of renting or owning their own houses. Categories 1 and 2 receive government assistance. Households that consume more than or equal to RWF 159,375 are classified in Ubudehe Category 3 and 4 [15]. These categories are not considered poor and do not receive government assistance. Category 3 includes wealthy farmers and investors while Category 4 includes large business owners and highly ranked government officials [16]. For health insurance, only community-based health insurance (Mutuelle), la Rwandaise Assurance Maladie (RAMA), and Medical Military Insurance (MMI) were categorized separately; all other insurances were categorized as "private insurances". The RAMA health insurance is a privately operated health insurance available to individuals formally employed in the both the public and private sectors. Marital status was categorized into three groups of married, single and widowed, separated or divorced. HIV status as well as comorbidities and exposures to HCV risk factors were self-reported. Self-reported comorbidities assessed included high blood pressure (HBP), diabetes, chronic renal failure (CRF), cancer, tuberculosis (TB). Risk factors assessed that were associated with parenteral routes of transmission included history of health facility-based surgical operation, traditional surgical operation and transfusion. Traditional surgical operation practices are defined as scarifications, male circumcision, tattoos, traditional dental extraction or uvulectomy done by a community member or traditional practitioner. Other risk factors assessed were number of sexual partners and self-reported presence of diagnosed viral hepatitis in a family member.

\section{Statistical methods \& data analysis}

Data was de-identified and after data cleaning, data were transferred and analyzed using SPSS version 20.0. Pearson Chi-square tests were used to test for association between HCVAb seroprevalence and other categorical or binary variables. Potential determinants of $\mathrm{HCV}$ infection were assessed in bivariate and multivariable models using logistic regressions with HCVAb seroprevalence as the outcome variable. Multivariate analysis was used to determine socio-demographic, behavioral and clinical factors that were independently associated with HCVAb Seroprevalence.

All variables in bivariate analyses were considered for inclusion in multivariate regression model if their inclusion was conceptually logical. Variables that were not significant were eliminated using backward stepwise method, variables were removed based on $P$-value producing a final model that could determine independent association between variables and $\mathrm{HCV}$ infection. Interaction between age and comorbidities were checked in the final multivariate model.

\section{Ethics}

The RBC of HIV/AIDS, STIs and Other Blood Borne Infections maintains the routinely collected program data analyzed for this study. The Medical Research Council of Rwanda governed the ethical procedures for the collection of these data and we obtained the authorization from the Ministry of Health to host the activities at different sites. The approval for utilization of data was obtained by Rwanda Biomedical Center (No 2407/RBC/2019).

\section{Results \\ Study population}

The total number of individuals screened was 327,383; $326,263(99.7 \%)$ received their results of which 22,183 (6.8\%) screened positive for HCVAb. There were 1120 $(0.3 \%)$ individuals without results due to drop-out??, insufficient blood sample, or indeterminate result. Sociodemographic characteristics of the study population are shown in Table 1 . The mean age of participants was 44.8 years. There were $145,537(45.8 \%)$ individuals older than 45 years of age, 224,382 (68.8\%) whom were female, and 247,437 (77.5\%) whom were married. 149,092 (45.5\%) were in Ubudehe category 3 . The vast majority of participants 303,206 (93.6\%) were using communitybased health insurance (Mutuelle). The province with the highest number of individuals screened was Eastern Province where 110,337 (35.0\%) of participants were screened.

Table 2 shows self-reported clinical characteristics and historical exposures of participants. Among comorbidities, 1867 (0.6\%) reported diabetes, 9510 (2.9\%) reported high blood pressure (HBP), 578 (0.2\%) reported chronic renal failure (CRF), 293 (0.2\%) reported cancer, 6597 (2\%) reported HIV-positive status, and 338 (0.1\%) reported a history of tuberculosis. Among self-reported historical exposures to risk factors, 17,876 (5.6\%) were surgically operated upon at least once, 5350 (1.6\%) received a blood transfusion at least once, 54,097 (16.6\%) had history of a traditional surgical practice, 8755 (2.7\%) had multiple sex partners (either current or historical), and 6190 (1.9\%) had a family member diagnosed with 
Table 1 General characteristics of participants

\begin{tabular}{|c|c|c|c|}
\hline Characteristics & Number of participants(\%) & HCV Ab positive by Characteristics(\%) & HCV Ab negative by Characteristics(\%) \\
\hline \multicolumn{4}{|l|}{ Gender $(N=326,175)$} \\
\hline Female & $224,382(68.8)$ & $15,431(6.9)$ & $208,951(93.1)$ \\
\hline Male & $101,793(31.2)$ & $6651(6.6)$ & $95,142(93.4)$ \\
\hline \multicolumn{4}{|l|}{$\operatorname{Age} \operatorname{group}(N=317,431)$} \\
\hline$<35$ years old & $92,243(29.1)$ & $3396(3.7)$ & $88,847(96.3)$ \\
\hline 35-44 years old & $79,651(25.1)$ & $3427(4.3)$ & $76,224(95.7)$ \\
\hline $45-54$ years old & $61,905(19.5)$ & $3516(5.7)$ & $58,389(94.3)$ \\
\hline $55-64$ years old & $50,556(15.9)$ & $4674(9.3)$ & $45,882(90.7)$ \\
\hline 65 years old and above & $33,076(10.4)$ & $6458(19.6)$ & $26,618(80.4)$ \\
\hline \multicolumn{4}{|l|}{ Marital status $(N=319,302)$} \\
\hline Married & $247,437(77.5)$ & $1030(3.7)$ & $23,713(96.3)$ \\
\hline Single & $28,188(8.8)$ & $15,266(6.2)$ & $12,922(93.8)$ \\
\hline Widow, Divorced and Separated & $43,677(13.7)$ & $4866(11.2)$ & $38,811(88.8)$ \\
\hline \multicolumn{4}{|l|}{ Ubudehe category $(N=318,855)$} \\
\hline Category 1 & $49,886(15.6)$ & $4462(9.0)$ & $45,424(91.0)$ \\
\hline Category 2 & $119,643(36.5)$ & $8034(6.7)$ & $111,609(93.3)$ \\
\hline Category 3 & $149,092(45.5)$ & $9097(6.1)$ & 139,995 (93.9) \\
\hline Category 4 & $234(0.1)$ & $17(7.3)$ & $217(92.7)$ \\
\hline \multicolumn{4}{|l|}{ Health Insurance $(N=323,953)$} \\
\hline Mutuelle & $303,206(93.6)$ & $21,095(7.0)$ & $282,111(93.0)$ \\
\hline RAMA & $14,708(4.5)$ & $515(3.5)$ & $14,193(96.5)$ \\
\hline MMl & $2574(0.8)$ & $78(3.0)$ & $2496(97.0)$ \\
\hline Other private insurances & $3465(1.1)$ & $218(6.3)$ & $3247(93.7)$ \\
\hline \multicolumn{4}{|l|}{ Province of screening $(N=315,040)$} \\
\hline East & $110,337(35.0)$ & $3313(5.0)$ & $107,024(95.0)$ \\
\hline North & $65,824(20.9)$ & $5277(5.9)$ & $60,547(94.1)$ \\
\hline West & $89,885(28.5)$ & $5880(12.0)$ & $84,005(88.0)$ \\
\hline South & 48,994 (15.6) & $7264(6.6)$ & $41,730(93.4)$ \\
\hline
\end{tabular}

viral hepatitis. Among all variables, no variables had > $5 \%$ missing values.

In the multivariate analysis as shown in Table 3 , being a male was statistically associated with HCVAb Seroprevalence [adjusted-OR $(\mathrm{aOR})=1.06,95 \% \mathrm{CI}:(1.03-1.10)]$. Age greater than 65 years old $[\mathrm{aOR}=4.87,95 \% \mathrm{CI}:(4.62-5.11)]$, and widowed/ separated and divorced marital status $[\mathrm{aOR}=1.36$, 95\%CI:(1.26-1.47)] were both associated with HCV Ab Seroprevalence. Having RAMA as health insurance had lower odds of HCVAb seroprevalence compared to those on Community based health insurance [aOR $=0.78,95 \% \mathrm{CI}:(0.70$ 0.85)] and being in Ubudehe Category 3 and 4 had lower odds of HCVAB seroprevalence than being in Ubudehe category $1[\mathrm{aOR}=0.9295 \% \mathrm{CI}:(0.88-0.96)]$. Relative to the Northern Province, all other provinces had a significantly higher seroprevalence in the Western Province, Southern Province and Eastern Province [aOR $=1.37,95 \% \mathrm{CI}$ :(1.311.43)]. Self-reported co-morbidities found to be associated with HCVAb prevalence included high blood pressure $(\mathrm{aOR}=1.95 \% \mathrm{CI}:(1.13-1.31)]$ and $\mathrm{CRF}(\mathrm{aOR}=1.29,95 \% \mathrm{CI}$ : (1.16-1.44)]. Self-reported risk factors associated with HCVAb prevalence were history of traditional surgical practices $(\mathrm{aOR}=1.09,95 \% \mathrm{CI}:(1.05-1.14)]$ and history of a family member diagnosed with viral hepatitis $(\mathrm{aOR}=1.27,95 \% \mathrm{CI}$ : $(1.15-1.40)]$.

\section{Discussion}

This study is the first national-level study in Rwanda to assess risk factors for HCV using the country's seroprevalence survey for $\mathrm{HCV}$ exposure (HCVAb). The national-level coverage and large sample size contribute to the strength of our findings. To our knowledge this is the first study to assess risk factors associated with HCVAb prevalence in a nationallyrepresentative screening program for members of the general population in SSA. 
Table 2 Distribution of potential self-reported risk factors for hepatitis $C$ that were assessed in the analysis

\begin{tabular}{|c|c|c|c|}
\hline Characteristics & Number of participants(\%) & HCV Ab positive by Characteristics(\%) & HCV Ab negative by Characteristics(\%) \\
\hline \multicolumn{4}{|c|}{ Suffering from Diabetes $(N=326,926)$} \\
\hline No & $325,059(99.4)$ & $21,961(6.8)$ & $303,098(93.2)$ \\
\hline Yes & $1867(0.6)$ & $182(9.8)$ & $1685(91.2)$ \\
\hline \multicolumn{4}{|c|}{ Suffering from HBP $(N=326,931)$} \\
\hline No & $317,421(97.1)$ & $21,054(6.7)$ & $296,367(93.3)$ \\
\hline Yes & $9510(2.9)$ & $1090(11.5)$ & $8420(88.5)$ \\
\hline \multicolumn{4}{|c|}{ Suffering from $\operatorname{CRF}(N=326,930)$} \\
\hline No & $326,323(99.8)$ & $21,692(6.7)$ & $10,940(93.3)$ \\
\hline Yes & $578(0.2)$ & $452(10.9)$ & $126(89.1)$ \\
\hline \multicolumn{4}{|c|}{ Suffering from Cancer $(N=326,901)$} \\
\hline No & $32,016(99.8)$ & $22,079(6.8)$ & 9937 (93.2) \\
\hline Yes & $293(0.2)$ & $63(10.9)$ & $230(89.1)$ \\
\hline \multicolumn{4}{|c|}{ HIV status $(N=326,913)$} \\
\hline Negative & $320,316(98.0)$ & $21,673(6.8)$ & 298,643 (93.2) \\
\hline Yes & $6597(2.0)$ & $470(7.1)$ & $6127(92.9)$ \\
\hline \multicolumn{4}{|c|}{ Ever had TB(N=326,919) } \\
\hline No & $326,581(99.9)$ & $22,108(6.8)$ & $304,473(93.2)$ \\
\hline Yes & $338(0.1)$ & $36(10.7)$ & $302(89.3)$ \\
\hline \multicolumn{4}{|c|}{ Ever been operated $(N=326,913)$} \\
\hline No & $309,037(94.4)$ & $20,916(6.8)$ & $288,121(93.2)$ \\
\hline Yes & $17,876(5.6)$ & $1225(6.9)$ & $16,651(93.1)$ \\
\hline \multicolumn{4}{|c|}{ Ever been transfused $(N=326,922)$} \\
\hline No & 321,572 & $21,691(6.8)$ & 299,881 (93.2) \\
\hline Yes & $5350(1.6)$ & $452(8.5)$ & $4898(91.5)$ \\
\hline \multicolumn{4}{|c|}{ Traditional operation and scarification $(N=326,652)$} \\
\hline No & $272,555(83.4)$ & $18,047(6.6)$ & $254,508(93.4)$ \\
\hline Yes & $54,097(16.6)$ & $4089(7.6)$ & $50,008(92.4)$ \\
\hline \multicolumn{4}{|c|}{ Having more than 1 sexual partner $(N=326,785)$} \\
\hline No & $318,030(97.3)$ & $21,601(6.8)$ & $296,429(93.2)$ \\
\hline Yes & $8755(2.7)$ & $538(6.2)$ & $8217(93.8)$ \\
\hline \multicolumn{4}{|c|}{ Viral Hepatitis in the family $(N=326,882)$} \\
\hline No & $320,692(98.1)$ & $21,609(6.8)$ & 299,083 (93.2) \\
\hline Yes & $6190(1.9)$ & $531(8.6)$ & 5659 (91.4) \\
\hline
\end{tabular}

Compared to previous studies conducted in Rwanda among PLHIV and pregnant women that produced prevalence estimates of 4.6 and $2.6 \%$ respectively $[9,10]$, the HCVAb seroprevalence estimated by this study is $6.8 \%$. The likely explanation for this higher prevalence is the strategic decision by the campaign to target older individuals and the self-selected nature of voluntary participants where individuals who had reason to suspect that they had viral hepatitis may have come forward for testing. Factors associated with being HCVAb positive following adjusted analysis included older age with $0.1 \%$ of risk of developing HCV every year for each participant, lower socioeconomic status, geographic variation, family history of $\mathrm{HCV}$ and exposures to traditional surgical operations. Associations between HCV infection and both family history of $\mathrm{HCV}$ and exposures to traditional surgical operations remained significant after adjusting for age.

Traditional scarifications and operations, though heavily discouraged, are still widely prevalent in informal healthcare practices in Rwanda. Although data on frequency of traditional surgical practices in Rwanda is limited, the Rwanda Demographic Health Survey 2015 reported that $8.5 \%$ of circumcisions for people between 
Table 3 Prevalence of HCV according to different characteristics among participants

\begin{tabular}{|c|c|c|c|c|c|c|c|c|}
\hline \multirow[t]{2}{*}{ Characteristics } & \multirow{2}{*}{$\begin{array}{l}\text { Number of } \\
\text { participants }\end{array}$} & \multirow{2}{*}{$\begin{array}{l}\text { HCV } \\
\text { positive(\%) }\end{array}$} & \multicolumn{3}{|c|}{ Bivariate analysis } & \multicolumn{3}{|c|}{ Multi-variable analysis } \\
\hline & & & $\overline{\mathrm{OR}}$ & $(95 \% \mathrm{Cl})$ & $P$-value & $\mathrm{OR}$ & $(95 \% \mathrm{Cl})$ & $P$-value \\
\hline \multicolumn{9}{|l|}{ Gender $(N=325,078)$} \\
\hline Female & 223,627 & $15,431(6.9)$ & 1 & & & 1 & & \\
\hline Male & 101,451 & $6651(6.6)$ & 0.95 & $(0.92-0.98)$ & $<0.001$ & 1.06 & $(1.03-1.10)$ & $<0.001$ \\
\hline \multicolumn{9}{|l|}{ Age $\operatorname{group}(N=316,336)$} \\
\hline$<35$ years old & 91,922 & $3396(3.7)$ & 1 & & & 1 & & \\
\hline 35-44 years old & 79,337 & $3427(4.3)$ & 1.18 & $(1.12-1.24)$ & $<0.001$ & 1.11 & $(1.05-1.17)$ & $<0.001$ \\
\hline 45-54 years old & 61,733 & $3516(5.7)$ & 1.57 & $(1.50-1.65)$ & $<0.001$ & 1.30 & $(1.23-1.37)$ & $<0.001$ \\
\hline 55-64 years old & 50,379 & $4674(9.3)$ & 2.67 & $(2.55-2.79)$ & $<0.001$ & 2.11 & $(2.00-2.22)$ & $<0.001$ \\
\hline 65 years old and above & 32,965 & $6458(19.6)$ & 6.35 & $(6.08-6.64)$ & $<0.001$ & 4.86 & $(4.62-5.11)$ & $<0.001$ \\
\hline \multicolumn{9}{|l|}{ Marital status $(N=318,211)$} \\
\hline Single & 28,105 & $1030(3.7)$ & 1 & & & 1 & & \\
\hline Married & 246,593 & $15,266(6.2)$ & 1.74 & $(1.63-1.85)$ & $<0.001$ & 1.152 & $(1.07-1.24)$ & $<0.001$ \\
\hline Widow, separated and divorced & 43,513 & $4866(11.2)$ & 3.31 & $(3.09-3.55)$ & $<0.001$ & 1.36 & $(1.26-1.47)$ & $<0.001$ \\
\hline \multicolumn{9}{|l|}{ Ubudehe category $(N=318,784)$} \\
\hline Category 1 & 49,767 & $4462(9.0)$ & 1 & & & 1 & & \\
\hline Category 2 & 119,347 & $8034(6.7)$ & 0.73 & $(0.71-0.76)$ & $<0.001$ & 0.97 & $(0.93-1.01)$ & 0.112 \\
\hline Category 3 and 4 & 148,686 & $9114(6.1)$ & 0.66 & $(0.64-0.69)$ & $<0.001$ & 0.92 & $(0.88-0.96)$ & $<0.001$ \\
\hline \multicolumn{9}{|l|}{ Health Insurance $(N=322,856)$} \\
\hline Mutuelle & 302,152 & $21,095(7.0)$ & 1 & & & 1 & & \\
\hline RAMA & 14,673 & $515(3.5)$ & 0.49 & $(0.44-0.53)$ & $<0.001$ & 0.775 & $(0.70-0.85)$ & $<0.001$ \\
\hline $\mathrm{MMl}$ & 2570 & $78(3.0)$ & 0.42 & $(0.33-0.52)$ & $<0.001$ & 0.804 & $(0.63-1.02)$ & 0.08 \\
\hline Other private insurances & 3436 & $218(6.3)$ & 0.90 & $(0.78-1.03)$ & 0.12 & 1.06 & $(0.91-1.23)$ & 0.47 \\
\hline \multicolumn{9}{|l|}{ Province of screening $(N=313,945)$} \\
\hline North & 65,804 & $3313(5.0)$ & 1 & & & 1 & & \\
\hline West & 89,767 & $5277(5.9)$ & 1.18 & $(1.13-1.23)$ & $<0.001$ & 1.19 & $(1.14-1.25)$ & $<0.001$ \\
\hline South & 48,976 & $5880(12.0)$ & 2.57 & $(2.46-2.69)$ & $<0.001$ & 1.98 & $(1.88-2.08)$ & $<0.001$ \\
\hline East & 109,398 & $7264(6.6)$ & 1.34 & $(1.40-1.50)$ & $<0.001$ & 1.37 & $(1.31-1.43)$ & $<0.001$ \\
\hline \multicolumn{9}{|l|}{ Suffering from Diabetes $(N=325,829)$} \\
\hline No & 323,971 & $21,961(6.8)$ & 1.00 & & & & & \\
\hline Yes & 1858 & $182(9.8)$ & 1.49 & $(1.28-1.74)$ & $<0.001$ & & & \\
\hline \multicolumn{9}{|l|}{ Suffering from HBP $(N=325,834)$} \\
\hline No & 316,372 & $21,054(6.7)$ & 1.00 & & & 1.00 & & \\
\hline Yes & 9462 & $1090(11.5)$ & 1.83 & $(1.71-1.95)$ & $<0.001$ & 1.22 & $(1.13-1.31)$ & $<0.001$ \\
\hline \multicolumn{9}{|l|}{ Suffering from $\operatorname{CRF}(N=325,833)$} \\
\hline No & 321,698 & $21,692(6.7)$ & 1.00 & & & 1.00 & & \\
\hline Yes & 4135 & $452(10.9)$ & 1.68 & $(1.54-1.87)$ & $<0.001$ & 1.29 & $(1.16-1.44)$ & $<0.001$ \\
\hline \multicolumn{9}{|l|}{ Suffering from Cancer $(N=325,804)$} \\
\hline No & 325,228 & $22,079(6.8)$ & 1.00 & & & & & \\
\hline Yes & 513 & $63(10.9)$ & 1.69 & $(1.30-2.19)$ & $<0.001$ & & & \\
\hline \multicolumn{9}{|l|}{ HIV status $(N=325,816)$} \\
\hline Negative & 319,234 & $21,673(6.8)$ & 1.00 & & & 1 & & \\
\hline Yes & 6582 & $470(7.1)$ & 1.06 & $(0.96-1.16)$ & 0.26 & 1.08 & $(1.05-1.32)$ & 0.001 \\
\hline
\end{tabular}

Ever had $\operatorname{TBC}(N=325,822)$ 
Table 3 Prevalence of HCV according to different characteristics among participants (Continued)

\begin{tabular}{|c|c|c|c|c|c|c|c|c|}
\hline \multirow[t]{2}{*}{ Characteristics } & \multirow{2}{*}{$\begin{array}{l}\text { Number of } \\
\text { participants }\end{array}$} & \multirow{2}{*}{$\begin{array}{l}\mathrm{HCV} \\
\text { positive(\%) }\end{array}$} & \multicolumn{3}{|c|}{ Bivariate analysis } & \multicolumn{3}{|c|}{ Multi-variable analysis } \\
\hline & & & $\mathrm{OR}$ & $(95 \% \mathrm{Cl})$ & $P$-value & OR & $(95 \% \mathrm{Cl})$ & $P$-value \\
\hline No & 325,484 & $22,108(6.8)$ & 1.00 & & & & & \\
\hline Yes & 338 & $36(10.7)$ & 1.636 & $(1.16-2.31)$ & 0.005 & & & \\
\hline \multicolumn{9}{|c|}{ Ever been operated $(N=325,816)$} \\
\hline No & 307,980 & $20,916(6.8)$ & 1.00 & & & & & \\
\hline Yes & 17,836 & $1225(6.9)$ & 1.01 & $(0.95-1.07)$ & 0.69 & & & \\
\hline \multicolumn{9}{|c|}{ Ever been transfused $(N=325,825)$} \\
\hline No & 320,488 & $21,691(6.8)$ & 1.00 & & & & & \\
\hline Yes & 5337 & $452(8.5)$ & 1.28 & $(1.16-1.41)$ & $<0.001$ & & & \\
\hline \multicolumn{9}{|c|}{ Traditional operation and scarifications $(N=325,555)$} \\
\hline No & 271,590 & $18,047(6.6)$ & 1.00 & & & 1.00 & & \\
\hline Yes & 53,965 & $4089(7.6)$ & 1.15 & $(1.11-1.19)$ & $<0.001$ & 1.09 & $(1.05-1.14)$ & $<0.001$ \\
\hline \multicolumn{9}{|c|}{ Viral Hepatitis in the family $(N=325,785)$} \\
\hline No & 319,616 & $21,609(6.8)$ & 1.00 & & & 1.00 & & \\
\hline Yes & 6169 & $531(8.6)$ & 1.30 & $(1.19-1.42)$ & $<0.001$ & 1.27 & $(1.15-1.40)$ & $<0.001$ \\
\hline \multicolumn{9}{|c|}{ Interaction Age_HBP(N=326,263) } \\
\hline$<35$ years old & 317,891 & $21,669(6.4)$ & 1 & & & 1 & & \\
\hline 35-44 years old & 1418 & $80(5.6)$ & 0.84 & $(0.67-1.05)$ & 0.12 & 1.14 & $(0.80-1.61)$ & 0.46 \\
\hline $45-54$ years old & 1880 & $111(5.9)$ & 0.88 & $(0.73-1.07)$ & 0.19 & 1.27 & $(1.01-1.61)$ & 0.05 \\
\hline 55-64 years old & 2606 & $276(10.6)$ & 1.66 & $(1.46-1.83)$ & $<0.001$ & 1.04 & $(0.85-1.27)$ & 0.71 \\
\hline 65 years old and above & 2468 & $574(22.2)$ & 3.99 & $(3.62-4.39)$ & $<0.001$ & 1.18 & $(1.06-1.30)$ & 0.002 \\
\hline \multicolumn{9}{|c|}{ Interaction Age_CRF $(N=326,263)$} \\
\hline$<35$ years old & 322,909 & $21,788(3.7)$ & 1 & & & 1 & & \\
\hline $35-44$ years old & 767 & $48(6.3)$ & 0.13 & $(0.09-0.20)$ & $<0.001$ & 1.32 & $(0.97-1.81)$ & 0.08 \\
\hline 45-54 years old & 765 & $56(7.3)$ & 0.21 & $(0.15-0.29)$ & $<0.001$ & 1.38 & $(1.01-1.61)$ & 0.03 \\
\hline $55-64$ years old & 1069 & $129(12.1)$ & 0.25 & $(0.18-0.34)$ & $<0.001$ & 1.26 & $(1.04-1.54)$ & 0.02 \\
\hline 65 years old and above & 753 & $183(24.3)$ & 0.43 & $(0.33-0.55)$ & $<0.001$ & 1.24 & $(1.03-1.50)$ & 0.02 \\
\hline \multicolumn{9}{|c|}{ Interaction Age_HIV $(N=326,263)$} \\
\hline$<35$ years old & 321,031 & $21,801(6.8)$ & 1 & & & 1 & & \\
\hline 35-44 years old & 1940 & $119(6.1)$ & 1.00 & $(0.80-1.26)$ & 0.97 & 1.36 & $(1.12-1.65)$ & 0.00 \\
\hline $45-54$ years old & 1920 & $101(5.3)$ & 0.76 & $(0.62-0.93)$ & 0.01 & 0.89 & $(0.73-1.10)$ & 0.29 \\
\hline 55-64 years old & 1023 & $92(9.0)$ & 1.36 & $(1.09-1.68)$ & 0.01 & 0.93 & $(0.75-1.16)$ & 0.53 \\
\hline 65 years old and above & 349 & $70(20.1)$ & 3.44 & $(2.65-4.48)$ & $<0.001$ & 1.01 & $(0.77-1.32)$ & 0.94 \\
\hline
\end{tabular}

15 and 59 years old were performed by traditional practitioners or a family friend [17]. Collectively, these findings suggest a need for interventions targeted to traditional healers such as increasing population awareness on the risks of traditional cuttings for infectious disease transmission. The Ministry of Health and RBC strongly advise individuals in Rwanda to seek health advice from official health facilities rather than traditional healers. Our findings add further evidence to the potential harm of unregulated traditional practices.

Although the most frequently discussed transmission routes for $\mathrm{HCV}$ are parenteral, there has also been much debate on the impact of household exposure on $\mathrm{HCV}$ transmission [18]. Our study found an association between familial history of viral hepatitis and HCVAb prevalence. Previous observational studies have reported clustering of cases within households and documented evidence of higher disease prevalence among individuals with an infected family member compared to the general population [19-22]. Moreover, a recent cross-sectional survey of $\mathrm{HCV}$ patients conducted in China showed that long term exposure to an infected family member was associated with infection [23], an indication that a constant exposure to low-risk transmission routes such as 
razors, tooth brushes and nail clippers could still contribute to infection. However, another plausible explanation for our findings is that members of the same household could be exposed to the same external risk factors. For example, family members visiting the same traditional medicine practitioner with unsafe needle practices may have an elevated risk for infection. Given low awareness among the population on potential risk of transmission of $\mathrm{HCV}$ within households, patients with HCV should be counselled on prevention of disease transmission to their cohabitants. Cohabitants should be offered the option of receiving $\mathrm{HCV}$ counseling and undergoing $\mathrm{HCV}$ testing.

Similar to other studies conducted in Rwanda on people living with HIV and on pregnant women $[9,10]$, older age showed strong associations with HCVAb with a trend of higher odds of infection with increasing age group (Fig. 1). It is likely that older individuals are more likely to have historical exposure to risk factors such as unhygienic medical procedures and scarifications, either within a health facility or with traditional practitioners, prior to implementation of current infection control policies.

Co-morbidities that showed associations with HCVAb prevalence were HBP and CRF. According a large household survey in Rwanda, 33.2\% of people aged 55-64 years old had high blood pressure while $6.7 \%$ of people between 25 and 34 years old had high blood pressure [24]. There might be interaction between co-morbidities and age (see Table 3). CRF could be a complication of chronic HCV and HCV can be acquired during different procedures performed by health care providers during management of CRF, such as dialysis [25]. Self-reported HIV infection was associated with HCVAb prevalence and can be explained by potential shared modes of transmission between HIV and HCV.
Individuals in socioeconomic category of Ubudehe category 3 have lower odds of HCVAb compared to individuals in the lowest socioeconomic category of Ubuduhe category 1 . Individuals using RAMA as health insurance compared to those using Mutuelle also have lower odds of infection. Individuals from lower socioeconomic categories may be at a higher risk for $\mathrm{HCV}$ due to more exposures to unhygienic practices in informal health settings or sharing of sharps such as razors.

This study also found that people from the Northern province have lower Seroprevalence for HCV compared to other provinces. This may be due to the fact that other provinces have higher migration across borders and a greater refugee population. Apart from socio-economic status, other unmeasured cultural practices or risk factors could have contributed to these geographical differences [10, 11, 13, 23, 26].

Several limitations were identified. The demographic profile of the sample population of voluntary participants differed from the Rwandan population at large, with a substantially greater proportion of females (69\%) and a higher median age (43.0) than the general population. Thus, the prevalence estimate and risk factors found to be associated with HCVAb may not be generalizable to the entire population. Since children are less likely to be infected with $\mathrm{HCV}$, the prevalence of $\mathrm{HCV}$ in Rwanda is likely lower than reported in this study. As this study utilized presence of anti-HCV antibody as the primary marker of $\mathrm{HCV}$ infection, the risk factors identified are relevant for present or previous $\mathrm{HCV}$ infection and may not be associated with chronic viremic state. Other unmeasured risk factors such as exposure to mass casualties through war and conflict, sexual violence, refugee status, occupational risk like health care providers or community based traditional practices

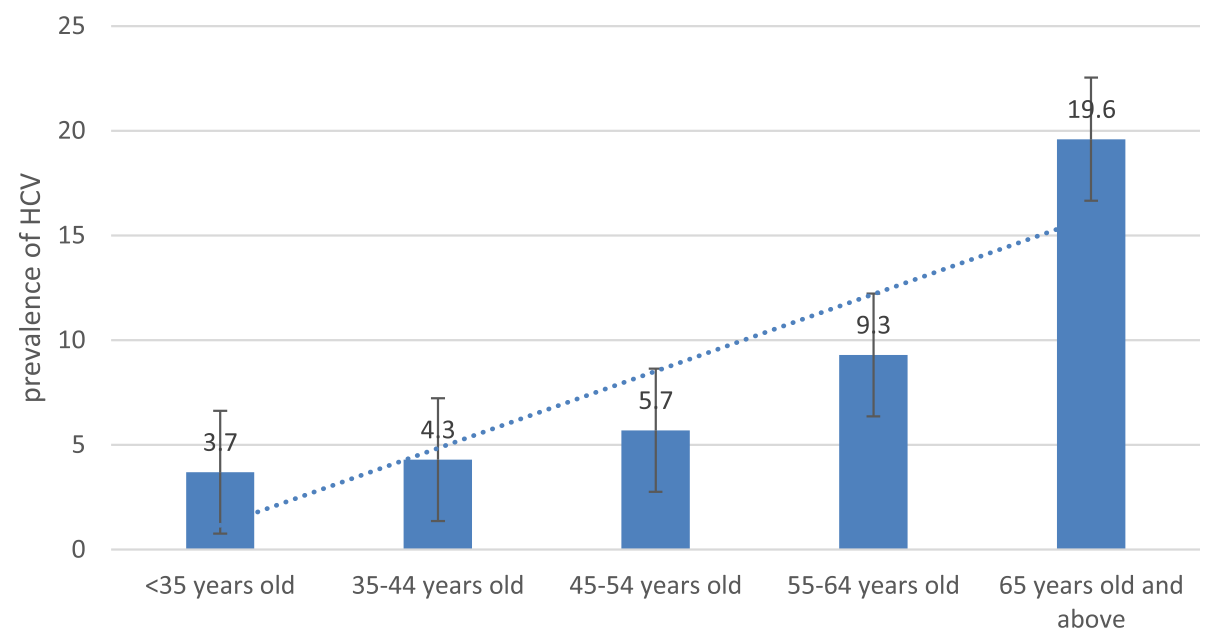

Age group

Fig. 1 Prevalence of HCVAb by age category of participants 
may have been more prevalent historically. Key populations, such as injection drug users and men who have sex with men, were not specifically identified or characterized in this study, though the proportion of individuals with these risk factors have been previously reported to be low in the Rwandan general population [27]. Lastly, this study relied on routinely collected data and self-report to assess clinical variables (e.g. HIV status) or historical exposure which may have led to misclassification. Participants are likely to have underreported at random with respect to the outcome due to poor recall of historical events. If a true association exists between $\mathrm{HCV}$ infection and variables identified in this study, then random misclassification of exposures would have led odds ratio towards the null. If exposures were recorded accurately, we would expect to obtain the true effect of the known risk factors on HCVAb. Also plausible is that individuals with lower health literacy had lower awareness of risk factors. These individuals could have had either higher risk for $\mathrm{HCV}$ infection due to more exposures to less hygienic health practices or lower risk for $\mathrm{HCV}$ due to less healthcare-seeking overall.

\section{Conclusion}

$\mathrm{HCV}$ is a worldwide epidemic that can cause death and severe liver disease complications especially in low- and middle-income countries and has been identified as a public health problem in Sub-Saharan Africa. The high HCVAb seroprevalence found in this study reiterates the importance of addressing the $\mathrm{HCV}$ burden in both Rwanda and in sub-Saharan Africa. Screening should be universal and priority for testing and screening should be given to people who are at high risk such as older age, lower socioeconomic status, geographic variation, history of traditional surgical practices, and family exposures for both prevention and screening as Rwanda commits to achieving WHO targets of eliminating HCV by 2030.

\section{Abbreviations \\ Cl: Confidence Interval; CRF: Chronic Renal Failure; ELISA: Enzyme-Linked Immunosorbent Assays; HBV: hepatitis B virus; HCV Ab: Hepatitis C Virus Antibody; HCV: Hepatitis C Virus; HIV/AIDS: Human Immunodeficiency virus/ Acquired Immunodeficiency Diseases Syndrome; MEDIPLAN: Private insurance initiated by Insurance Company called SORAS; MINALOC: Ministry of Local Government; MMI: Military Medical Insurance; OR: Odd Ratio; PLHIV: People Living with HIV; RAMA: La Rwandaise Assurance Maladie: Health insurances for employees of public and private sectors; RBC: Rwanda Biomedical Center; SPSS: Statistical Package for the Social Sciences; TB: Tuberculosis; WHO: World Health Organization}

\section{Acknowledgements}

We especially thank the participants and health care providers from hospitals and health centers where screening took place, as this study would not have been possible without them. We also thank the Ministry of Health and Rwanda Biomedical Center (RBC) and Ministry of Health leaders especially Dr. Jeannine U. Condo, Director General of RBC and Dr. Jean Pierre Nyemazi, Permanent Secretary in the Ministry of Health and other RBC staff for their generous assistance and support.

\section{Authors' contributions}

MJD, CJL, NCK, UJ, SU, DD, DSM, JS, MS, NMP, NG, MH and SN developed the study protocol and study design. MJD, CJL, NCK, UJ, SU, DD and JS collected the data. MJD did the data analysis and developed the figure and tables. All authors participated in interpretation of the data. MJD did the literature review and wrote the first draft of the manuscript. All authors reviewed and provided critique of the manuscript. All authors read and approved the final manuscript.

\section{Funding}

The screening of hepatitis C virus was funded by the Government of Rwanda. The funder has no direct involvement in study design and data collection. The design of the study, analysis, interpretation of data and writing the manuscript were conducted independently by authors.

\section{Availability of data and materials}

The datasets generated during the current study are not publicly available but are available from the corresponding author on reasonable request.

\section{Ethics approval and consent to participate}

The ethical procedures for the collection of these data were governed by the Medical Research Council of Rwanda and site authorizations were obtained from the Ministry of Health for hosting sites. Secondary analyses of routinely collected data are exempt by the Rwanda Biomedical Center. The approval for utilization of data was obtained by Rwanda Biomedical Center (No 2407/RBC/2019).

Consent for publication

Not Applicable.

\section{Competing interests}

This study used routine data collected by Rwanda Biomedical Center. The authors declare that they have no competing interests.

\section{Author details}

${ }^{1}$ IHDPC Department, Rwanda Biomedical Center, Po Box 7162, Kigali, Rwanda. ${ }^{2}$ Health Department, Clinton Health Access Initiative (CHAl), Kigali, Rwanda. ${ }^{3}$ School of Population and Public Health, University of British Columbia, Vancouver, Canada. ${ }^{4}$ Division of Global Health Equity, Brigham \& Women's Hospital, Boston, USA. ${ }^{5}$ Department of Infectious Disease at the Alfred Hospital and Infectious Diseases Epidemiology, Monash University in Melbourne, Melbourne, Australia.

Received: 10 May 2019 Accepted: 26 July 2019

Published online: 05 August 2019

\section{References}

1. World Health Organization. Global hepatitis report, 2017. (2017). doi: ISBN 978-92-4-156545-5

2. Bartenschlager $\mathrm{R}$, et al. Critical challenges and emerging opportunities in hepatitis $C$ virus research in an era of potent antiviral therapy: considerations for scientists and funding agencies. Virus Res. 2018;248: 53-62.

3. Petruzziello A, Marigliano S, Loquercio G, Cozzolino A, Cacciapuoti C. Global epidemiology of hepatitis $C$ virus infection: an up-date of the distribution and circulation of hepatitis C virus genotypes. World J Gastroenterol. 2016; 22:7824-40

4. Cornberg $\mathrm{M}$, et al. A systematic review of hepatitis $C$ virus epidemiology in Europe, Canada and Israel; 2011. p. 30-60. https://doi.org/10.1111/j.14 78-3231.2011.02539.x.

5. Shiferaw F, Letebo M, Bane A. Chronic viral hepatitis: policy, regulation, and strategies for its control and elimination in Ethiopia. BMC Public Health. 2016:16:1-13.

6. Karoney MJ, Siika AM. Hepatitis C virus ( HCV ) infection in Africa : a review. Pan Afr Med J. 2013:8688:1-8.

7. Eze JC, et al. Prevalence and risk factors for hepatitis $\mathrm{C}$ and human immunodeficiency virus coinfection among children in Enugu Nigeria. Afr J Infect Dis. 2014;8:5-8.

8. Semugoma NP, et al. Hepatitis C: a south African literature review and results from a burden of disease study among a cohort of drug-using men 
who have sex with men in Cape Town, South Africa. S Afr Med J. 2017;107: 1116.

9. Mutagoma M, et al. Hepatitis C virus and HIV co-infection among pregnant women in Rwanda. BMC Infect Dis. 2017;17:4-9.

10. Umutesi J, et al. Prevalence of hepatitis $B$ and $C$ infection in persons living with HIV enrolled in care in Rwanda. BMC Infect Dis. 2017;17:1-7.

11. Huang $C$, et al. Prevalence and risk factors of hepatitis $C$ among former blood donors in rural China. Int J Infect Dis. 2012;16:e731-4.

12. Hayes MO, Harkness GA. Body piercing as a risk factor for viral hepatitis: an integrative research review. Am J Infect Control. 2001;29:271-4.

13. Gorar ZA, Butt ZA, Aziz I. Risk factors for bloodborne viral hepatitis in healthcare workers of Pakistan: a population based case-control study. BMJ Open. 2014:4:1-7.

14. National Institute of Statistics of Rwanda (NISR), M. of F. and E. P. (MINECOFIN) [Rwanda]. Rwanda Household, Integrated Survey, Living Conditions. (2015)

15. Nizeyimana P, Lee K-W, Sim S. A study on the classfication of households in Rwanda based on factor scores. J Korean Data Inf Sci Sociaty. 2018;29:547-55.

16. National Institute of Statistics of Rwanda (NISR) [Rwanda]. Rwanda Integrated Household (EICV) 2013/2014. (2015).

17. National Institute of Statistics of Rwanda (NISR) [Rwanda], Ministry of Health (MOH) [Rwanda], and I. I. Rwanda Demographic and Health Survey 2014-15. Rockville: (NISR, MOH,and ICF International); 2015.

18. Hajiani E, Masjedizadeh R, Hashemi J, Azmi M, Rajabi T. Hepatitis C virus transmission and its risk factors within families of patients infected with hepatitis C virus in southern Iran: Khuzestan. World J Gastroenterol. 2006;12: $7025-8$

19. Zekry Farg I, El-Dien Nouh M, AlFateh Mahrous O. Intrafamilial transmission of hepatitis C virus in Menoufia governorate. Menoufia Med J. 2014;27:478.

20. Amran B, Lankarani M, Ardebili M, Sepehrimanesh M, Nejabat MA, Hemmati Rad SYH. Evaluation of hepatitis C virus intrafamilial transmission among in Iran. Gastroenterol Hepatol Bed Bench. 2016;9:250-8.

21. Omar MZ, et al. Role of intrafamilial transmission in high prevalence of hepatitis C virus in Egypt. Hepatic Med Evid Res. 2017;9:27-33.

22. Paez Jimenez A, et al. HCV iatrogenic and intrafamilial transmission in greater Cairo, Egypt. Gut. 2010;59:1554-60.

23. Luo B-f, Hui-ying Rao YG, W L. Risk factors for familial clustering of hepatitis C virus infection in a Chinese Han population: a cross-sectional study. BMC Public Health. 2018;18:708.

24. Rwanda Ministry of Health, W. H. O. Rwanda non-communicable diseases risk factors report. Rockville: WHO; 2015.

25. Latt N, Alachkar N, Gurakar A. Hepatitis C virus and its renal manifestations: a review and update. Gastroenterol Hepatol. 2012;8:434-45.

26. Mohamed HI, et al. Hepatitis C, hepatitis B and HIV infection among Egyptian prisoners: Seroprevalence, risk factors and related chronic liver diseases. J Infect Public Health. 2013;6:186-95.

27. Rwanda Biomedical Center/Institute of HIV/AIDS, Disease Prevention and Control Department (RBC/IHDPC), S. \& of Public Health (SPH), UNAIDS, and I. I. Estimating the Size of Populations through a Household Survey. (2012).

\section{Publisher's Note}

Springer Nature remains neutral with regard to jurisdictional claims in published maps and institutional affiliations.

Ready to submit your research? Choose BMC and benefit from:

- fast, convenient online submission

- thorough peer review by experienced researchers in your field

- rapid publication on acceptance

- support for research data, including large and complex data types

- gold Open Access which fosters wider collaboration and increased citations

- maximum visibility for your research: over $100 \mathrm{M}$ website views per year

At $\mathrm{BMC}$, research is always in progress.

Learn more biomedcentral.com/submissions 